\title{
A Theoretical Analysis of Cumulative Sum Slope (CUSUM-Slope) Statistic for Detecting Signal Onset (begin) and Offset (end) Trends from Back- ground Noise Level
}

\author{
David Tam*
}

Department of Biological Sciences, University of North Texas, Denton, Texas 76203, USA

\begin{abstract}
A theoretical analysis of the cumulative sum (CUSUM) technique for detecting a series of time signals from noisy background is provided. The statistic using CUSUM-slope is introduced as a measure for capturing the average of signals within the time-window, in which the slope is computed. This provides a time-independent method for estimating the signal content within the time-window. The detection criterion is provided for different window-lengths. The results showed that this CUSUM-slope statistic is highly sensitive to the detection of subtle hidden trends in the data sequence with noise filtered even in very low signal-to-noise environment.
\end{abstract}

Key Words: Cumulative sum, CUSUM, Signal detection, Noise filtering, Serial dependence, Trend analysis, Time-series analysis.

\section{INTRODUCTION}

Extracting signal from background noise is one of the most common problems faced in statistical analysis, especially in detection of changes in a data sequence from the random fluctuation of background noise. In this paper, we will limit the definition of noise to events that are generated by a random process, while signals that are events that generated by non-random processes. Thus, noise in this context is usually generated from a stationary process that has a zero-mean, while signal has a non-zero mean. The statistical task is to identify the events that are generated from the nonrandom process in the midst of the background noise generated from a random process.

Specifically, when the unknown signal is embedded in background noise, the task is to identify the signal by removing the background noise. If the signal-to-noise ratio is large, it is a trivial problem. But if the signal-to-noise ratio is low (especially when background noise is an order of magnitude bigger than the signal), then it is a daunting task to extract signal from noise when the signal is "buried" in the background noise. This definition of signal and noise is also the classical definition used in engineering and industrial quality control in defining signal-to-noise ratio as a measure of meaningful information (non-random signal) compared to background noise (random signal). No other assumptions about the putative signal or noise are made except for the non-random and random processes under consideration, respectively.

For example, in electrical engineering, the statics in radio transmission is considered as noise, whereas the sound transmitted is considered as signal. Similarly, in industrial

\footnotetext{
*Address correspondence to this author at the Department of Biological Sciences, University of North Texas, Denton, Texas 76203, USA;

E-mail:dtam@unt.edu
}

quality control for detecting failure of components in assembly line, the random variability in components sampled at control point is considered as noise due to statistical fluctuations, whereas if the components consistently failed when sampled at control point that is considered as signal (i.e., change from random background fluctuations). Deriving a sensitive measure for detecting changes in the presence of background fluctuations would provide a good quality control criterion for product safety in manufacturing.

Since we usually do not have any a priori knowledge of the underlying non-random process in most practical situations, we would not know what the signal might be if we were to design a detection method for such as unknown signal. Furthermore, statistical methods are often used to detect and deduce the unknown underlying process in spite of the variability in the data sequence. Thus, it is non-essential to define "signal", in this statistical analysis, as long as the signal is generated by a non-random process, which is different from the "noise" that is generated by a random process.

Finding a sensitive statistical measure to detect such nonrandom process is particularly important in many real-world applications when the presumed relevant signal generated is unknown prior to the analysis. Examples include brain (neural) signals recorded in experiments, using fMRI (functional magnetic resonance imaging) and EEG (electroencephalogram) techniques, where the signal-to-noise ratio is extremely low and the presumed signal generated by the brain is unknown prior to the experiment.

Furthermore, when a time-series signal is considered, it is often essential to detect trends (i.e., sequential changes) when the signal appears and when it disappears from the background noise. Stock market fluctuation is a good example to show how trend analysis can help delineate the daily fluctuation in a volatile market from the underlying trend of market crash or economic recovery (non-random process). 
Thus, the detection of the onset time of appearance of signal (i.e., deviation from background noise) and the offset time of the disappearance of signal with statistical significance is important in signal trend analysis when the signal is unknown. This poses a difficult problem to extract the signal when [1] the signal is unknown in the first place, and [2] the signal-to-noise ratio is very small, i.e., when the putative signal is much smaller than the random fluctuation of background noise. In other words, we seek to detect non-random events in amidst of a random process.

More precisely, signal in this definition is the deviation from the background noise. In other words, we make no assumptions about the signals, except that they deviate from background noise. For instance, in the search of possible intelligent intergalactic communication from outer space, we would not know what that signal might be, except that the signal would be different from the background radiation noise in the universe.

\section{CLASSICAL CUMULATIVE SUM (CUSUM) TECH- NIQUE}

One of the traditional statistical techniques to detect significant changes in a data sequence from its random background noise is the cumulative sum (CUSUM) test introduced by Page [1], in 1954. It is used to detect sequential changes in a sequence of data points in a discrete-time random process. Most often, a time-series is considered in CUSUM analysis, which can be applied to any data sequence without loss of generality.

Many different statistical detection methods, using the CUSUM technique, have been developed to establish the statistical significance criteria for detection of signal onset, such as CUSUM-charts [2-10], cumulative observedexpected (O-E) plots [11], and resetting sequential probability ratio (RSPRT) charts [12-14]. In particular, these CUSUM statistics were widely used in detecting onset of deviation in sequential data in areas such as quality control [15-17] as well as the medical fields [11,14,18-21]. Various statistical significance tests have been applied to CUSUM analysis to detect significant deviations from the mean by the CUSUM-chart method, using the V-mask form of CUSUM $[16,22]$, which depends on run length [23-26].

The most commonly used CUSUM-chart [2-10] procedure is a statistical technique for the detection of changes from the mean level of a series of sequential data points. Since, by definition of background noise, the statistical mean of the noise is zero and constant. On the contrary, the "signal" is defined as the deviation from this constant mean. Thus, the CUSUM procedure, when applied appropriately, is very sensitive to changes that are small compared to the fluctuation of individual data points from its mean.

Since most of the above CUSUM statistics rely on sample run history in the detection of signal from background noise, the statistical measures used are dependent on the cumulative sum of the total signal and noise since the beginning of the data sample, which is precisely the definition of CUSUM - cumulative sum of the signal plus noise since the beginning of time. Because of the dependence on the time origin of the data sample, statistical measures derived from the cumulative sum are also dependent on the selection of the specific time origin of the data sample during the analysis and the entire prior sequential history of the data sample by definition. In essence, CUSUM is a statistical measure to detect the serial-dependence of the signal in the presence of background noise since the beginning of time.

\section{ALTERNATE ORIGIN-INDEPENDENT CUSUM METHOD}

In this paper, we propose to use an alternate statistical method to detect the time of signal onset that is independent of the specific sample time origin, thus, independent of the cumulative run history, unlike the other CUSUM statistics. In other words, we seek to eliminate the dependence on the selection of time origin in the statistical criteria. Developing the statistical detection method that is independent of its cumulative history since the beginning of time could theoretically eliminate the accumulation of serial-dependence that is dependent on the selection of a particular time origin in the data set for analysis.

In this paper, we will prove that the "slope" of the CUSUM (CUSUM-slope) is a statistical measure that estimates the "average signal content" (without the background noise) during the interval (time-window), over which the CUSUM-slope is computed [27]. This CUSUM-slope measure also represents the trend of signals (i.e., how they deviate from the background noise). It can also be used to detect the onset (beginning), and offset (end) of the signal, and the duration of such signal. Furthermore, we will prove that the CUSUM-slope value is statistically measured that estimates the normalized algebraic sum of all signals within that timewindow, in which the CUSUM-slope is computed.

The statistical decision criteria are also given in this paper to determine the signal onset time, i.e., the time that the data sequence starts to deviate significantly from the mean background noise level. Provision of statistical criteria are especially important for determining the onset time of changes when the signal-to-noise ratio is very small (i.e., for determining the time of signal onset when background noise is much greater than the signal itself).

\section{BRIEF REVIEW OF THE CUSUM METHOD}

Suppose we have a set of discrete sequential data points $\left\{x_{-n}, \ldots, x_{0}, x_{1}, x_{2}, \ldots, x_{n},\right\}$, where $n$ is a positive integer (see Fig. 1). Without any loss of generality, this sequence of data points can be considered as a time-series, where $n$ denotes the time of occurrence of the data point. The cumulative sum (CUSUM) at time $t$ is given by $[1,2]$

$$
c_{t}=\sum_{i=1}^{t} x_{i}
$$

Note that the CUSUM $c_{t}$ is monotonically increasing function where $x_{i}$ are non-negative (if $x_{i} \geq 0$ ), such as a counting process (see Fig. 2). Note that the CUSUM in Fig. (2) is essentially a measure of the area under the curve in Fig. (1). It is intuitively obvious that if there is a deviation (change) in the data sequence from the background noise level, the slope of such monotonically increasing curve would change (with a steeper or shallower slope). It will be shown below that the traditional CUSUM-chart plot method 
uses a horizontal V-mask envelope to capture such change in slope in the CUSUM curve as the statistical significance criterion.

In general, $c_{t}$ is not necessarily monotonically increasing if $x_{i}$ are real numbers. Furthermore, $c_{t}$ is serially dependent on the prior signal content from the selected time origin to sample interval of interest, i.e., between time 1 and $t$. If a different time origin, $l$, is used, and different run length, $t$, is used, then the computed CUSUM will be different because it will include different segments of signal such that:

$$
c_{t}=\sum_{i=l}^{t+l-1} x_{i}
$$

Thus, the choice of time origin and the run length in computing the CUSUM is essential. It can affect the result because if the signal varies over time (i.e., non-constant), then the CUSUM measure would not be time-independent, but dependent on the choice of the selected time origin and the length of cumulated prior history.

\section{CUSUM-CHART PLOT DETECTION METHOD}

One of the statistical detection criteria proposed was the CUSUM-chart plot method [2-10], in which the "mean path" of the sum is given by:

$$
C_{t}=\sum_{i=1}^{t}\left(x_{i}-k\right)
$$

where $k$ is a constant. It is used to detect positive drift in the process parameter, in this case, the observed mean is $k$. This CUSUM-chart plot will take a turn upward if the process mean increases above $k$. Thus, it is a one-sided test for positive deviations above the mean.

If $x_{i}-k<0$, the path of CUSUM-chart plot will point downwards, indicating that there is no positive deviation. The CUSUM-chart method will reset the time origin if $C_{t}$ falls below zero so as to detect positive deviations only in this one-sided test [3]. The duration from time-origin to the reset-point is called the "run length" in CUSUM-chart plot analysis. Thus, the run length is highly dependent on the data segment under consideration, and varies depending on not only the particular data sequence but also the time-origin (i.e., $i=l$ in Eq. 1 b) used as the reference point that "slides" along the $x$-axis and resets by the above criterion.

The statistical detection criterion in CUSUM-chart plot is set by an "alarm value", $h$, in which $C_{t}>h$ will trigger an alarm as significant deviation from the mean. Thus, the statistical detection criteria in CUSUM-chart plot is given by:

$$
C_{t}>h
$$

where $h$ is a predefined (most often, an $a d$ hoc) criterion. If the process is known beforehand in both of its normal target state and all its possible departure states, it would be possible to derive the optimum value for $h$ mathematically. But full knowledge of the underlying process is rarely known in reality without any a priori knowledge. Thus, most CUSUMchart detection methods provide an estimate of $h$ for a given "average run length" (A.R.L.), $t$.
In order to provide a two-sided test for the positive and negative deviations, then two one-sided schemes would be applied simultaneously to detect upward and downward deviations of the mean path. When the process exceeds this threshold $h$, it marks the time of deviation at $t_{a}$ :

$$
t_{a}=\min \left\{k: R_{t} \geq h\right\}
$$

where

$$
R_{t}=\max _{j \leq t} \sum_{i=1}^{j}\left(x_{i}-\mu\right)-\min _{j \leq t} \sum_{i=1}^{j}\left(x_{i}-\mu\right)
$$

and $\mu$ represents the expected mean.

\section{CUSUM-CHART V-MASK METHOD}

To overcome those limitations, the traditional CUSUMchart V-mask was introduced [2] such that a horizontal-V is used as an envelope, sliding along the $C_{t}$ curve, for the charting method to detect both positive and negative deviation simultaneously, such that if $C_{t}$ deviates/drifts beyond this $\mathrm{V}$ mask, then the detection alarm is set. More specifically, the $\mathrm{V}$-mask envelope is bounded by an angle $2 \theta$, with its vertex at the current point and at a distance $d$ from its horizontal axis. That is, $d$ is considered to be the "leading distance", and $\theta$ the angle between each of the arms of the horizontal $\mathrm{V}$-mask and the horizontal axis. The value of the detection criteria, $d$ and $\theta$, are often chosen arbitrarily from empirical trials. Assuming $\mu$ be the target mean of the process,

$$
C_{t}=\sum_{i=1}^{t}\left(x_{i}-\mu\right)
$$

then the difference between the observed mean, $k$, and target mean, $\mu$, would be given by:

$$
c=k-\mu=\rho(d+a) \tan \theta
$$

where $a$ is the horizontal distance between successive points on the chart, and $\rho$ is the scale-factor converting the vertical distance on the chart to $x$-units. Solving for the trigonometry of the control-chart V-mask envelope will yield,

$$
\rho y=\left(x_{t+1}-\mu\right) \sqrt{2 \cos \theta}
$$

as the detection threshold criterion for setting the alarm.

\section{CUSUM-SLOPE}

If one wants to detect local changes in signal content, the statistical variable used must be independent of the time origin. To achieve such time-independence, one could use the time-derivative of the CUSUM in Eq. (1a) as the statistical variable for detection of such changes:

$$
s_{t}=\frac{d c_{i}}{d t}
$$

For finite discrete variables, let us define the "slope" $\left(m_{t, \tau}\right)$ of the CUSUM between time $t$ and $t+\tau$ as an approximation of the time derivative in Eq. (9) by:

$$
m_{t, \tau}=\frac{c_{t+\tau}-c_{t}}{\tau}
$$


where $\tau$ is the time-window in the CUSUM-slope calculation. Rewriting Eq. (10) using the definition of the CUSUM in Eq. (1a) gives:

$$
m_{t, \tau}=\frac{1}{\tau} \sum_{i=t+1}^{t+\tau} x_{i}
$$

\section{SIGNAL AND NOISE CONTENT}

The data point $x_{t}$ at time $t$ can be considered as the sum of signal $\left(s_{t}\right)$ and noise $\left(n_{t}\right)$. Assuming the principle of superposition applies, i.e., linearality for additive noise,

$$
x_{t}=s_{t}+n_{t}
$$

In this case, without any loss of generality, we will define the background noise generated by a random process as data sequence that has a zero-mean over the interval of interest, $t$, i.e.,

$$
\bar{n}_{t}=\frac{1}{t} \sum_{i=1}^{t} n_{i} \cong 0
$$

for large $t$, and similarly, signal as the data sequence that has non-zero mean over the interval of interest, $t$, i.e.,

$$
\bar{s}_{t}=\frac{1}{t} \sum_{i=1}^{t} s_{i} \neq 0
$$

\section{CUSUM-SLOPE AS MOVING-AVERAGE OF THE "CAPTURED" SIGNAL WITH BACKGROUND NOISE REMOVED}

Decomposing the signal and noise in CUSUM-slope of Eq. (11) by Eq. (12), it becomes

$$
m_{t, \tau}=\frac{1}{\tau} \sum_{i=t+1}^{t+\tau}\left(s_{i}+n_{i}\right)=\frac{1}{\tau} \sum_{i=t}^{t+\tau} s_{i}+\frac{1}{\tau} \sum_{i=t}^{t+\tau} n_{i}
$$

By definition of noise in Eq. (13), the CUSUM-slope becomes

$$
m_{t, \tau} \cong \frac{1}{\tau} \sum_{i=t+1}^{t+\tau} s_{i}
$$

for a finite time-window, $\tau$. This shows that CUSUM-slope is approximately equal to the time average of the signal over a finite interval from $t$ and $t+\tau$, the time-window-of-interest. That is, the CUSUM-slope captures the signal content (the sum of signal with noise removed) within this time-window $\tau$. Thus, the CUSUM-slope between time $t$ and $t+\tau$ approximates the moving-average of the signal component of the data sequence between time $t$ and $t+\tau$ (starting at time origin t).

\section{INDEPENDENCE OF CUMULATIVE TIME HIS- TORY BY CUSUM-SLOPE METHOD}

The above analysis shows that CUSUM-slope estimates the signal content within this time-window $\tau$ independent of the prior history prior to time origin, $t$, for any $t$ in the data sequence. This moving-average provides history independence in the computation of the CUSUM statistics for estab- lishing significance criteria. This history independence is extremely important when the data sequence is serially dependent, and the signal onset time is unknown without any $a$ priori knowledge of what the signal is or when the signal starts.

\section{SIGNIFICANCE TEST FOR THE CUSUM-SLOPE}

The variance of the CUSUM-slope can be expressed in terms of original data set using Eq. (11) such that:

$$
\begin{aligned}
\operatorname{Var}\left(m_{t, \tau}\right) & =\operatorname{Var}\left(\frac{1}{\tau} \sum_{i=t+1}^{t+\tau} x_{i}\right) \\
& =\frac{1}{\tau^{2}} \sum_{i=t+1}^{t+\tau} \operatorname{Var}\left(x_{i}\right) \\
& =\frac{1}{\tau^{2}} \tau \sigma(x)^{2} \\
& =\frac{\sigma(x)^{2}}{\tau}
\end{aligned}
$$

where $\sigma$ represents the standard deviation. Thus, the variance of the CUSUM-slope, $\operatorname{Var}(m)$, is inversely proportional to window length, $\tau$,

$$
\operatorname{Var}\left(m_{t, \tau}\right) \propto \frac{1}{\tau}
$$

for a given data segment. That is, the variance of the CUSUM-slope is inversely proportional to the sample size, $\tau$, for computing the moving-average of the signal and noise. Thus, statistical criteria used to detect significant changes from the background noise level would also be inversely proportional to $\tau$, the sampling size used in the CUSUMslope calculation.

\section{NULL HYPOTHESIS}

For a special case, assume the data sequence contains that noise only, i.e., $x_{t}=n_{t}$ and $s_{t}=0$ (a null hypothesis). Then, Eq. (17) becomes:

$$
\begin{aligned}
\operatorname{Var}\left(m_{t, \tau}\right)_{\text {noise }} & =\operatorname{Var}\left(\frac{1}{\tau} \sum_{i=t+1}^{t+\tau} n_{i}\right) \\
& =\frac{\sigma(n)^{2}}{\tau}
\end{aligned}
$$

Since the variance of the noise, $\operatorname{Var}(n)$, is a constant for a given data segment of stationary noise, any deviation of the variance of the CUSUM-slope, $\operatorname{Var}(m)$, from this constant can be used to detect significant changes from the background noise. Note that the criterion used to detect significant changes is inversely proportional to $\tau$ (the time-window used in the calculation of the CUSUM-slope). Thus, statistical significance tests can be applied to the variance of the CUSUM-slope to determine the threshold criterion for onset time of the signal that is above and beyond the noise level.

Ideally, if a segment of data is known to contain noise only, it can be used as the "control" for determining the em- 
pirical mean and standard deviation of the background noise level. Thus, the observed mean of background noise prior to the CUSUM-slope analysis is given by:

$$
\bar{x}_{t}=\sum_{i=-t}^{0} \frac{x_{i}}{t}
$$

In some practical situations, this a priori knowledge of noise is known, such as in quality control where a predetermined data sequence contains only perfect components (without defects), or in neurophysiological experiment in which the data is collected in the resting state prior to the delivery of stimulus, these known data sequences can be considered as containing background noise only, i.e., without the signal of interest.

In other cases, if such background noise sequence is not known, then the current mean and standard deviation can be used as an approximation even though its mean level may be over or underestimated. In this case, the "control" statistic is estimating the deviation from the current level instead of deviation from the background noise level.

\section{FACTORS AFFECTING THE CHOICE OF TIME- WINDOW IN CUSUM-SLOPE METHOD}

As described above, an increase in the time-window $\tau$ is equivalent to increasing the sampling size in the movingaverage calculation. This not only increases the sample size, but also potentially includes more signals within this timewindow in the CUSUM-slope calculation. That is, the longer the time-window, the larger the sample size is used in the moving-average calculation; the more the observed mean will approach the expected mean of the signal. But since the variance of CUSUM-slope is inversely proportional to the time-window $\tau$ (see Eq. (18)), such an increase in timewindow length for computing CUSUM-slope would also reduce the level of significance in the criterion used for detecting changes from background noise level.

Furthermore, most importantly, the above is true only if the signal, $x_{i}$, continues to exist within this time-window, $\tau$. If the signal stops during this time-window, extending the sampling time-window would not yield extra statistical significance in the calculation, and (or estimation) of the observed signal mean. In fact, it will underestimate the observed signal mean when the signal stops, while the sampling size increases. This is because extending the timewindow after the signal had ceased would include only background noise (which has zero-mean), thus resulting in a smaller observed mean than the true mean of the signal in the moving-average (CUSUM-slope) calculation.

This shows that there is a realistic limit, in which expanding the time-window, $\tau$, may be helpful. If the signal continues to exist within the time-window, $\tau$, in the calculation of the moving-average represented by CUSUM-slope, then it would improve the statistic significance of the computed mean. If the signal ceases to exist within the extended timewindow, $\tau$, then it will result in an underestimation of the observed signal mean. That is to say, if the signal is stationary, increasing the sampling size would improve the statistical significance. If the signal is non-stationary, increasing the sampling size would potentially bias the observed mean, reducing the statistical significance of that estimated mean.

\section{CHOICE OF TIME-WINDOW IN CUSUM-SLOPE METHOD}

Thus, the best choice of $\tau$ used for finding the signal average is governed by various factors. If $s_{i}$ is a constant during an interval $[t, t+\tau]$, then the CUSUM-slope would be approximately constant, so the longer the time-window, $\tau$, taken for the calculation of CUSUM-slope, the more the observed mean will approach the expected mean of the signals.

But if $s_{i}$ is not constant (or non-stationary), then the choice of the time-window $\tau$ will be dictated by the duration in which signal is stationary locally. That is, if there is a subset of time-duration in which the signal is stationary locally (even though it may not be stationary globally), this timewindow can be used to enclose this local stationarity duration. For instance, in the analysis of daily temperature fluctuations, the temperature may show seasonal variations (global non-stationarity) but temperature may exhibit local stationarity over a shorter period within a season. Thus, the time-windows that span over multiple seasons will capture non-stationary signal whereas time-window that spans over the duration within a season can capture local stationary signal. In this case, piecewise stationarity can be obtained.

Consequently, the best choice of $\tau$ may assume a priori knowledge of the duration of signal, but in practice this difficulty may be overcome in some situations. For instance, let's say that the data sequence is a sinusoidal signal, using a time-window that corresponds to the period of the sinusoidal will yield a constant mean when sliding along the data sequence for computing the moving-average. If the timewindow used is longer (or shorter) than the period, then the CUSUM-slope (or the moving-average) will not be a constant when sliding along the data sequence. That is, if CUSUM-slope is a constant when sliding along the data sequence, the moving-average computed for the signal will also be a constant. If $m_{t, \tau}$ is a constant, then $\bar{S}_{t}$ is also a constant from Eq. (16) for a given $\tau$. Thus, the CUSUM-slope can be used to detect the finite period of local piecewise stationarity by using different $\tau$ in the calculation, and see which CUSUM-slope remains a constant.

\section{TWO-PASS DETECTION METHOD FOR DETER- MINING AN OPTIMAL TIME-WINDOW}

In general, one can determine the optimal $\tau$ and the best estimate of the duration of signal by a two-pass procedure. First, obtain the optimal $\tau$ by observing the constancy characteristic of the CUSUM-slope, $m_{t, \tau}$, as $\tau$ varies. Secondly, use the value of the $\tau$ selected above as the time-window in the calculation of the CUSUM-slope to determine the significant deviation of the CUSUM-slope above (or below) a certain statistical criterion level.

Since the observed mean and standard deviation of the CUSUM-slope are, in fact, corresponding to the mean and standard deviation of any sampled data (in moving-average calculation), classical statistical significance criteria can be used for detecting changes from the background noise level taking the consideration that such criteria is inversely proportional to the $\tau$ used.

\section{RESULTS}

We will apply the CUSUM-slope method to a sequence of data obtained from a pseudo-random number generator to 
illustrate the sensitivity of this signal detection method in trend analysis. In the first example, a sequence of uniformly distributed random data is generated by a widely-used software: Microsoft Excel (see Fig. 1). (Although other pseudorandom number generating algorithms, such as those provided by Numerical Recipe [28], could be used to generate pseudo-random sequences with lesser degree of serialdependence, we want to use this data set to illustrate the sensitivity of the CUSUM-slope method).

The corresponding CUSUM-chart is given in Fig. (2). The CUSUM curve in Fig. (2) essentially plots the area under the curve in Fig. (1). Because it is calculating the area under the curve, it is a monotonically increasing function, and the slope of the CUSUM-chart is almost a constant for this time-series for a random process. If the slope (diagonal line) becomes steeper or shallower, it usually indicates a change deviating from this random process. The divergence from this constant slope above (or below) the diagonal CUSUM-curve is used as the criterion for the traditional CUSUM-chart plot [2-10] to detect significant changes, in which an enveloping V-mask is used to delimit the threshold criteria for sounding an "alarm."

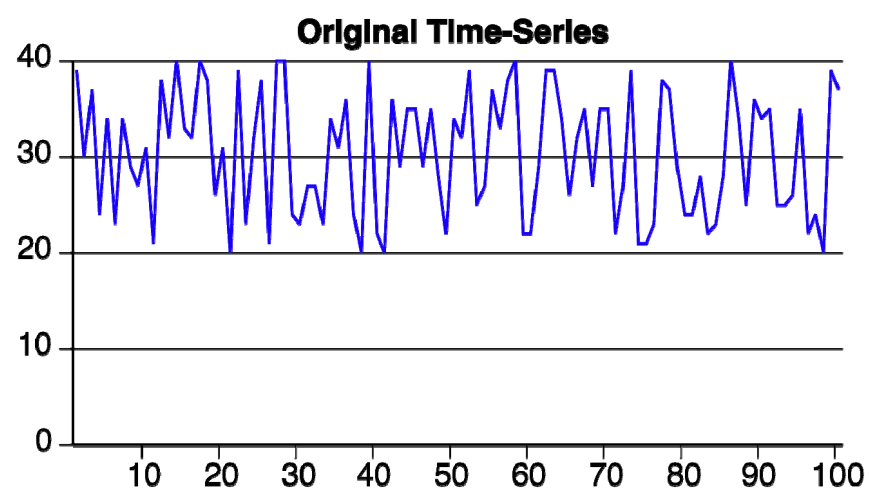

Fig. (1). Time-series of pseudo-random data generated by Microsoft Excel pseudo-random number generator.

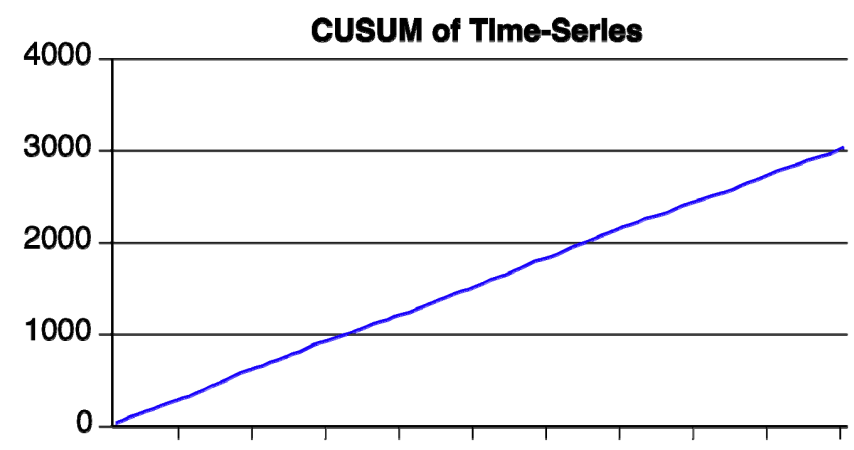

Fig. (2). CUSUM-chart plot of pseudo-random data in Fig. (1), showing the monotonically increasing characteristic of CUSUM chart method for a non-negative time-series of Fig. (1).

Figs. (3-5) show the CUSUM-slope plots with time-window $\tau=2,5$, and 10, respectively. Note that the CUSUMslope plots all capture the original pseudo-random data sequence with the noise removed. Different time-window length used in the CUSUM-slope yields different results. For short time-window $(\tau=2)$, it approximates the original data sequence (Fig. 3). For mid time-window ( $\tau=5$ ), it revealed the periodicity of the pseudo-random data sequence, showing approximately 9 periods in this sequence (Fig. 4). For longer time-window $(\tau=10)$, it revealed yet another periodicity of 2 periods in this same data sequence (Fig. 5).

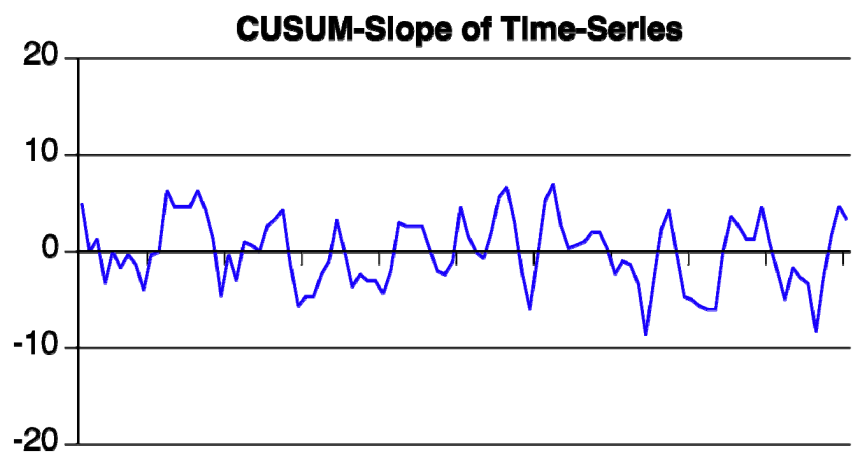

Fig. (3). CUSUM-slope plot of pseudo-random data in Fig. (1), using time-window, $\tau$, of 2 .

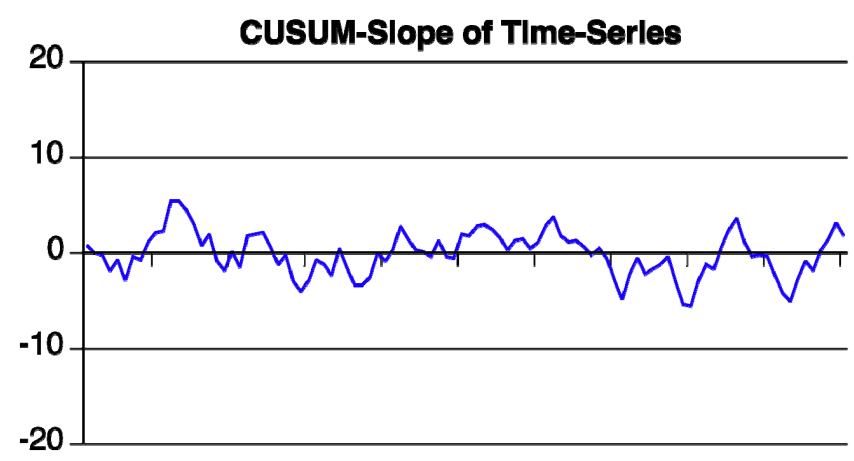

Fig. (4). CUSUM-slope plot of pseudo-random data in Fig. (1), using time-window, $\tau$, of 5, revealing serial-dependence (and periodicity) of the pseudo-random sequence.

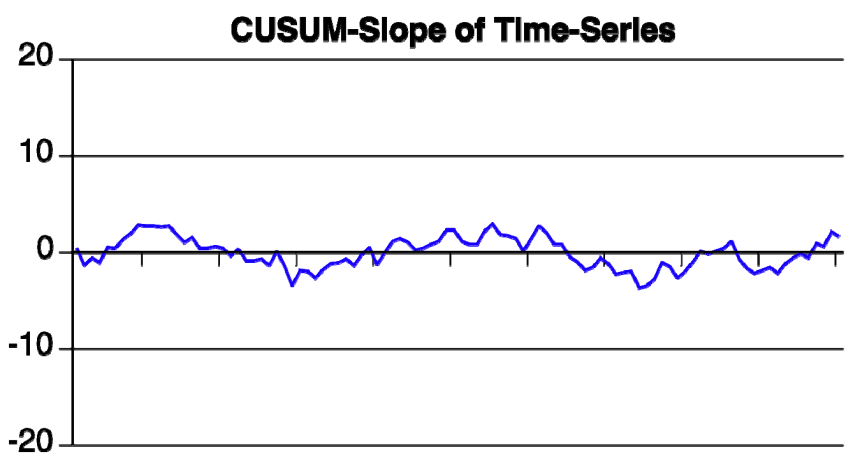

Fig. (5). CUSUM-slope plot of pseudo-random data in Fig. (1), using time-window, $\tau$, of 10, revealing serial-dependence (and periodicity) of the pseudo-random sequence, which has a longer period than that of Fig. (4).

This shows that the CUSUM-slope method not only detected that the pseudo-random number generator is periodic and serially dependent, but also repeated with different subperiodicities, i.e., a shorter period (in Fig. 4) and a longer period (in Fig. 5). Note that the CUSUM-slope automatically 
centers the data sequence around the zero-mean. Any deviation from this zero-mean can be used to test against the significance criterion for signal detection. Note also that since the $\operatorname{Var}\left(m_{t, \tau}\right)$ criterion is inversely proportional to $\tau$, the longer the time-window, $\tau$, the lower the criterion is needed to detect statistical significance.

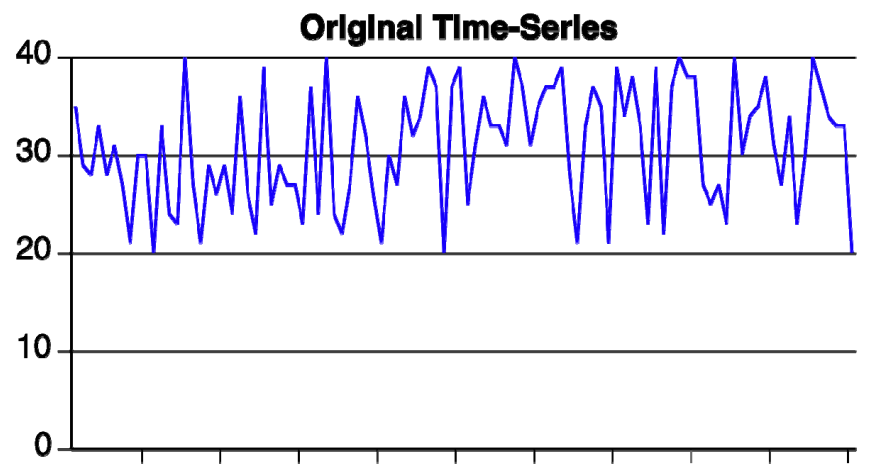

Fig. (6). Time-series of another pseudo-random data sequence generated from Microsoft Excel to compare the difference between the serial-dependence of the data sequences in Figs. (1 and $\mathbf{6})$.

In the second example, another pseudo-random number sequence is generated as shown in Fig. (6). In this case, the CUSUM-slope plots (Figs. 7-9) reveal similar underlying trends relatively independent of the time-window, $\tau$, used in computing the CUSUM-slope. This shows the data sequence in Fig. (6) is stationary for a longer duration than Fig. (1). In all cases, visual inspection reveals that the CUSUM-slope plots faithfully reproduced the original data sequence with the background noise filtered (or removed).

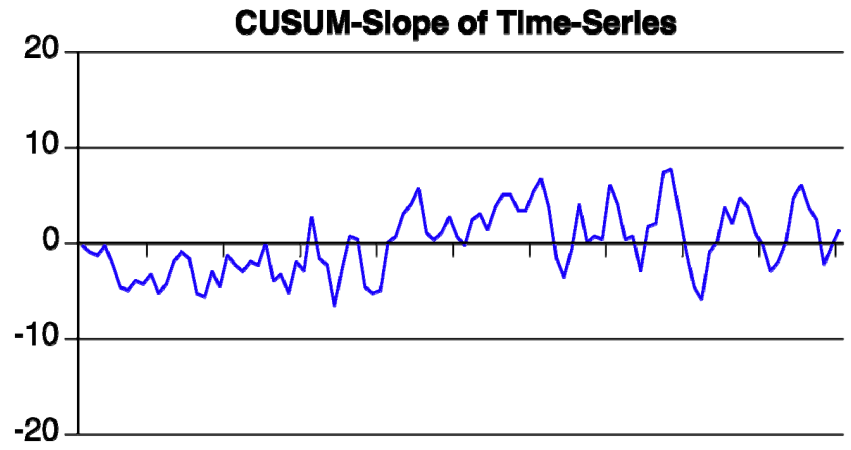

Fig. (7). CUSUM-slope plot of pseudo-random data in Fig. (6), using time-window, $\tau$, of 2 .

\section{DISCUSSION}

A novel detection method was introduced to augment the analysis of CUSUM using CUSUM-slope as a measure to estimate the signal content within a noisy background statistically. The CUSUM-slope essentially computes the timeaverage signal within the segment of data included in the CUSUM-slope calculation window without the noise, essentially corresponding to the moving-average calculation. This slope calculation enables the detection of signal anywhere along the data sequence, independent of the time-origin selected for the calculation of CUSUM.

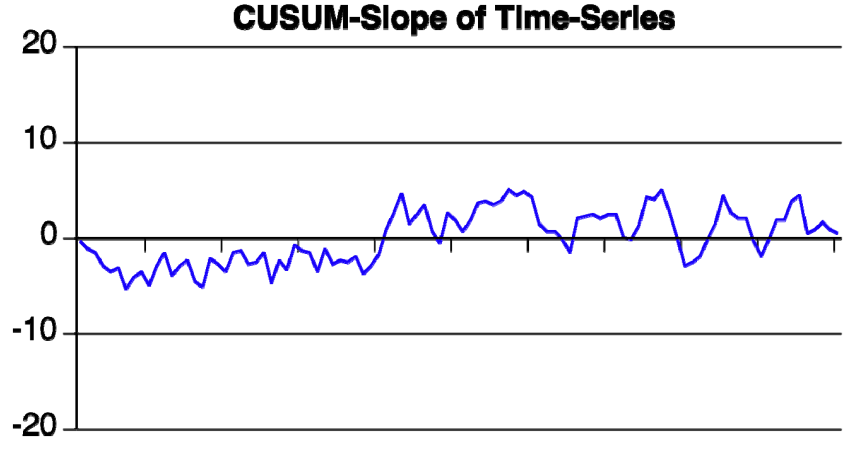

Fig. (8). CUSUM-slope plot of pseudo-random data in Fig. (6) using time-window, $\tau$, of 5 .

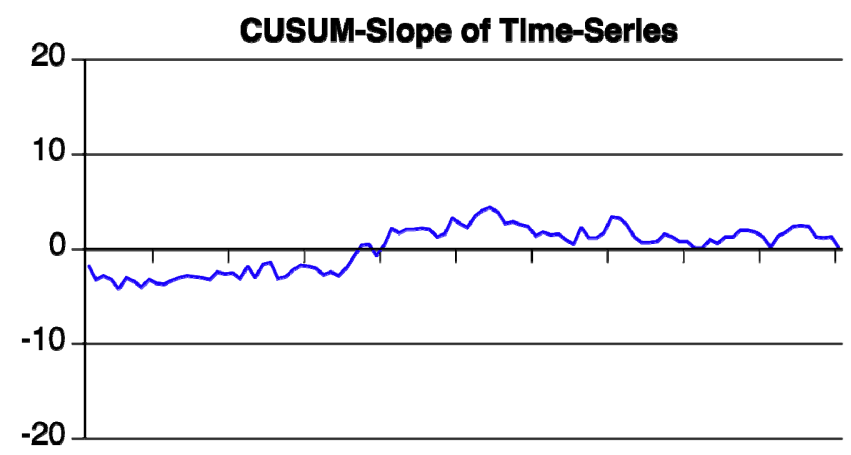

Fig. (9). CUSUM-slope plot of pseudo-random data in Fig. (6) using time-window, $\tau$, of 10 .

Because of this independence of time-origin, the detection statistic can be independent of the serial-dependence on its prior history, unlike CUSUM-chart detection method. Furthermore, no sliding V-mask envelope detection is needed to detect signals along the monotonically increasing CUSUM curve because of this time-origin independence. In fact, the CUSUM-slope is centered with a zero-mean horizontally rather than increasing monotonically, if a known background noise segment is used as the "control" for computing the observed mean of the background noise. A simple horizontal threshold crossing can be used as the statistical criterion for CUSUM-slope method rather than a sliding Vmask crossing for CUSUM-chart method.

The CUSUM-slope essentially captures the timeaveraged signal within the time-window $\tau$. The timeaveraged signal within that time-window is equal to the CUSUM-slope value $\left(m_{t, \tau}\right)$ at that point, starting at time $t$. This deviation from the zero-mean baseline noise level represents the signal that exceeds the background random fluctuations. Thus, the interpretation is much more straightforward than the CUSUM V-mask envelope chart method along the time-varying CUSUM-chart curve.

Furthermore, if the deviation of CUSUM-slope curve returns to the zero-mean level, it signifies that the signal disappears at that point, leaving only the noise behind. Such return to background noise level is not readily available in the V-mask method, since it only detects onset (beginning) of the signal but not the offset (end) of signal. If the 
CUSUM-slope deviates below the zero-mean level, it suggests that signal is removed from the noise in the negative direction, i.e., "inhibition" of signal rather than "excitation" of signal.

Extending this analysis can also be used to reveal trends in the data sequence, i.e., serial-dependence and serial correlation of the data. The pseudo-random number sequences illustrated above are examples to show how sensitive this CUSUM-slope method is in detecting the periodicity of the pseudo-random number generators, and trends in any data sequence. A true random number sequence would be revealed as a horizontal line with zero-mean for the CUSUMslope curve, but neither sequence in our example showed this characteristic. In fact, casual observation of the above two data sequence examples looked rather random and uniformly distributed, but the CUSUM-slope method revealed the taletelling characteristics of the pseudo-random sequence with either periodicity or sequential trend.

Although this CUSUM-slope method is similar to a band-pass filter operation using a moving-average, the difference is five-fold. First, the computed CUSUM-slope data sequence is centered at a zero-mean by definition of noise, which makes the statistical significance analysis much simpler than the CUSUM-chart statistical detection method using a V-mask envelope on the time-varying CUSUM curve such as Fig. (2). Second, the significant detection criterion is a constant for a given time-window, $\tau$, compared to the monotonically increasing criterion for the V-mask. Third, no sliding V-mask along the time-varying CUSUM-chart curve is needed to detect the significance level that requires a reset once it crosses the "alarm" level. The significance criterion is a simple horizontal line threshold crossing for the CUSUM-slope curve. Fourth, the signal detection criterion is independent of the selected time-origin for the analysis, whereas CUSUM-chart method is dependent on the selected time-origin in which CUSUM includes all prior signals (cumulative sum), by definition. Thus, the CUSUM-slope method can be applied to any arbitrary sequence of data at any time-origin without any dependence on the selected time-origin. The only dependence is the time-window length, $\tau$, used to compute the CUSUM-slope. Consequently, no reset of origin is needed, unlike the CUSUM-chart method that resets the origin producing different run-lengths in each reset. Fifth, the time-window in which the CUSUM-slope is calculated, can be used to capture the length (duration) of the signal or to detect the duration of local stationarity. The longer the window, the more data points will be included in the estimation of the observed signal mean. Assuming that the signal does not cease to exist within this time-window, this will increase the sampling size of the signal in the observed mean calculation. An iterative search method can be used to hunt for the signal duration with various windowlengths, $\tau$, to capture the maximal duration of the signal in the data sequence.

\section{SUMMARY}

A statistic based on the time derivative, or the "slope" of the CUSUM (CUSUM-slope), is introduced for the detection of signals in noisy background. Using the CUSUM-slope as the statistical variable, the trend, the time of occurrence and the duration of the signal component can be detected and quantified statistically. The time-averaged value of the signal within a time-window can also be obtained from CUSUMslope as a moving-average.

The criteria for detecting significant deviations from noise level can be expressed in terms of the standard deviation of the background noise and is inversely proportional to the value of $\tau$; the time-window used in the calculation of the CUSUM-slope.

\section{REFERENCES}

[1] E. S. Page, "Continuous inspection scheme", Biometrika, vol. 41, pp. $100-115,1954$

[2] G.A. Barnard, "Control charts and stochastic processes", J. Royal Stat. Soc. B (Methodological), vol. 21, pp. 239-71, 1959.

[3] E. S. Page, "Cumulative sum charts", Technometrics, vol. 3(1), pp. $1-9,1961$.

[4] P. L. Goldsmith and H. Whitfield, "Average run lengths in cumulative chart quality control schemes", Technometrics, vol. 3(1), pp. $11-20,1961$

[5] N. L. Johnson, "A simple theoretical approach to cumulative sum control charts”, J. Am. Stat. Assoc., vol. 56, 835-840, 1961.

[6] M. Basseville, and V.I. Nikiforov, Detection of Abrupt Changes: Theory and Application, Prentice-Hall, N.J: Englewood Cliffs, April 1993.

[7] S. H. Steiner, P. L. Geyer, and G. O. Wesolowsky, "Grouped data sequential probability ratio tests and cumulative sum control charts," Technometrics, vol. 38, pp. 230-237, 1996.

[8] S. H. Steiner, R. J. Cook, V. T. Farewell, and T. Treasure, "Monitoring surgical performance using risk-adjusted cumulative sum charts", Biostatistics, vol. 1, pp. 441-52, 2000.

[9] Chiu, W.K, “ The economic design of CUSUM charts for controlling normal means", Appl. Stat., vol. 23, pp. 420-433, 1974.

[10] Crosier, R.B, "A new two-sided cumulative sum quality control scheme", Technometrics, vol. 28, pp. 187-194, 1986.

[11] J. Poloniecki, O. Valencia, and P. Littlejohns, "Cumulative risk adjusted mortality chart for detecting changes in death rate: observational study of heart surgery", Br. Med. J., vol. 316, pp. $1697-$ $1700,1998$.

[12] A. Wald, "Sequential Analysis", New York: Wiley, 1947.

[13] A. Wald, "Sequential tests of statistical hypotheses", Ann. Math. Stat., vol. 16(2), pp. 117-86, 1945.

[14] O. A. Grigg, V. T. Farewell, and D. J. Spiegelhalter, "The use of risk-adjusted CUSUM and RSPRT charts for monitoring in medical Contexts", Stat. Meth. Med. Res., vol. 12, pp. 147-170, 2003.

[15] J.M. Lucas, and R.B Crosier, "Fast initial response for cusum quality-control schemes: give your cusum a head start", Technometrics, vol. 24(3), pp. 199-205, 1982.

[16] D.M. Hawkins, and D.H. Olwell, Cumulative sum charts and charting for quality improvement, New York: Springer, 1997.

[17] A. Nix, R.J. Rowlands, and K.W. Kemp, "Internal quality control in clinical chemistry", Stat. Med., vol. 6, pp. 425-440, 1986.

[18] S.H. Steiner, R.J. Cook, V.T. Farewell, and T. Treasure, "Monitoring paired binary surgical outcomes using cumulative sum charts", Stat. Med., vol. 18, pp. 69-86, 1999.

[19] J. Lipdki, E. R. Merrill, and W. Janczewski, "Analog calculation of cumulative sums from peri-stimulus time histograms and application of the technique to the analysis of rhythmic discharge of lung afferents", J. Neurosci. Methods, vol. 7, pp. 165-170, 1983.

[20] P. H. Ellaway, "An application of cumulative sum technique (cusums) to neurophysiology”, J. Physiol. (Lond.)., vol. 265, pp. 12P, 1977.

[21] P.H. Ellaway, "Cumulative sum technique and its application to the analysis of peristimulus time histograms", Electroenceph. Clin. Neurophysiol., vol. 45, pp. 302-304, 1978.

[22] G.A. Barnard, "Control charts and stochastic processes", J. Royal Stat. Soc., vol. 21, pp. 239-271, 1959.

[23] D. Brook, and D.A. Evans, "An approach to the probability distribution of cusum run length", Biometrika, vol. 3, pp. 539-549, 1972

[24] A.L. Goel, and S.M. Wu, "Determination of arl and a contour nomogram for cusum charts to control normal mean", Technometrics, vol. 13(2), pp. 221-230, 1971.

[25] R.A. Khan, "On cumulative sum procedures and the sprt with applications", J. Royal. Stat. Soc., 46(1), pp. 79-85, 1984. 
[26] E. Yashchin, "On a united approach to the analysis of two-sided cumulative sum control schemes with head starts", Adv. Appl. Probab., vol. 17, pp. 562-593, 1985.

[27] D. C. Tam, "Correlation of cerebellar Purkinje cell activity with the kinematics of a voluntary visually-guided closed-loop movement and interactions among Purkinje cells", Ph. D. Dissertation, 1987.
University of Minnesota, Minneapolis, University Microfilms International, Minnesota: Ann Arbor, 1987.

[28] W. H. Press, S. A. Teukolsky, W. T. Vetterling, and B. P. Flannery, Numerical Recipes: The Art of Scientific Computing, 3rd ed. New York: Cambridge University Press, 2007.

(C) David Tam; Licensee Bentham Open.

This is an open access article licensed under the terms of the Creative Commons Attribution Non-Commercial License (http://creativecommons.org/licenses/ by-nc/3.0/) which permits unrestricted, non-commercial use, distribution and reproduction in any medium, provided the work is properly cited. 